\title{
Unevenness of Loop Location in Complex Networks
}

\author{
An Zeng, Yanqing $\mathrm{Hu}$, Zengru $\mathrm{Di}^{*}$ \\ Department of Systems Science, School of Management and Center for Complexity Research, \\ Beijing Normal University, Beijing 100875, China
}

(Dated: November 13, 2018)

\begin{abstract}
The loop structure plays an important role in many aspects of complex networks and attracts much attention. Among the previous works, Bianconi et al find that real networks often have fewer short loops as compared to random models. In this paper, we focus on the uneven location of loops which makes some parts of the network rich while some other parts sparse in loops. We propose a node removing process to analyze the unevenness and find rich loop cores can exist in many real networks such as neural networks and food web networks. Finally, an index is presented to quantify the unevenness of loop location in complex networks.

PACS numbers: 89.75.Hc, 89.75.Da, 89.75.Fb
\end{abstract}

Introduction. In the last decade, the researches on complex networks have rapidly developed. At the same time, the loop structure has attracted much attention. Loops are very important in complex networks. They can not only characterize the detail structure of networks but also relate to the structural correlations, motifs, robustness and redundancy of pathways, and affect some dynamical as well as equilibrium critical phenomena of the networks[1,2]. Recently, to avoid the effect of the loop structure, some researchers even study the acyclic networks[3,4].

For the self-avoiding loop, researchers focus mainly on two aspects: the total number of loops and the the dynamic effect of the loop structure. In the former case, many counting methods have been proposed[5-11]. In undirected networks, short loops can be exactly counted in terms of powers of the adjacency matrix[8]. This method can not deal with long loops, because the counting equation will become very complicated when facing long loops. In directed networks, short loops can be estimated by using $N_{L} \simeq \frac{1}{L} \operatorname{Tr} A^{L}$ while long loops can be calculated by the entropy $[9,10]$. Moreover, some researchers analyze statistics of loops with different length $L$. They use the Monte Carlo sampling method to get the frequency and find that the loop number is sharply peaked around a characteristic loop length $L^{*}$. Also, they use $L^{*}$ and the relevant index to characterize the networks[11]. On the other hand, the dynamic effect of the loop structure has been studied frequently. It has been pointed out that the loop structure is related to the activity in neural networks such as self-sustained activities[12,13,14] and synchronization $[4,15,16]$. Specifically, the self-sustained activity can not survive without the loop structure and the synchronization will be weakened when emerging a dominant loop in the network. What's more, a scaling behavior of loops is used to explain some critical phenomenon in percolation[17] and loop number is also used as a ranking method to quantify the role of

*zdi@bnu.edu.cn both nodes and links[18].

However, many problems about loops still remain unnoticed. In the ref.[10], Bianconi, Gulbahce and Motter find that many real networks have fewer loops than the counterpart random networks which are a kind of random networks with the same number of in- and outlinks in each node as the real networks. Actually, the loops number in different parts of a network varies according to the function of the regions. For example, the feed-forward part of the neural networks are sparse of loops[3] while other parts in the brain need loops to carry out self-sustained oscillation for precessing information $[13,14]$. For the food web networks, the loops number in the metazoan part are relatively small while there are many short loops among the microorganisms, called microbial loops, for fixed carbon repacking and recovery path of ecosystem[19]. Obviously, loops locate unevenly in many real networks. Some communities of these networks will be rich in loops while loops will be sparse in other parts. This leads us to an interesting question: what is the detail organization of loops location like in the networks? In this paper, we focus on the unevenness of loops location. We first study the distribution of loops on single nodes. Then we analyze the rich loop core phenomenon of uneven loops location by a node removing process in some real networks. Finally, we propose an index to measure the unevenness.

Heterogenous distribution of loops on single nodes. In the first step, we should study the loops on each single node to help us understand how the loops locate in the network. For a given network with size $N$, if we want to obtain how many loops passing through a specific node, we can simply remove the node from the network and count how many loops decreases, the decrement is the number of loops on this node. As $N_{L}$ is the loops number with the length $L$ of a network, we denote the $\hat{N}_{L}(i)$ as the number of loops with length $L$ in the network after the node $i$ is removed. So the node has $C_{L}(i)=N_{L}-\hat{N}_{L}(i)$ loops with length $L$ passing through. The number of short loops in directed network can be expressed in terms of powers of the adjacency matrix. In particular, $N_{L} \simeq \frac{1}{L} \operatorname{Tr} A^{L}$, provided 
that $\kappa \equiv \max _{i} \sum_{j} \sum_{m}^{\prime}\left(\begin{array}{c}l \\ m\end{array}\right)\left|\lambda_{j}^{-m} P_{i j} P_{j+m, i}^{-1}\right| \ll 1[9]$. Because $C_{L}(i)=N_{L}-\hat{N}_{L}(i)$, we can count the short loops on single nodes in any networks.

Here, we focus on the distribution of $C_{L}(i)$ in different networks. We can compare $C_{L}^{r}(i)$ of real networks with $C_{L}^{c}(i)$ of the counterpart random networks and $C_{L}^{e}(i)$ of the corresponding ER random networks. The counterpart random networks are a kind of uncorrelated random networks with the same number of in- and out-links in each node as the real networks. The corresponding ER random networks is given with the same size and the total number of links as the real networks. Of course, $C_{L}^{r}(i)$, $C_{L}^{c}(i)$ and $C_{L}^{e}(i)$ can be obtained by $C_{L}(i)=N_{L}-\hat{N}_{L}(i)$. Actually, the expected value of $C_{L}^{c}(i)$ can be gained by the formula based on the degree sequence. Motivated by the formula of the expected number of loops in the uncorrelated random network $[10,20]$, we derive the expected number of loops on single nodes in undirected and directed random networks.

For undirected random networks, the expected number $E\left(N_{L}\right)$ of short loops with length $\mathrm{L}$ is given by[20]

$$
E\left(N_{L}\right)=\frac{1}{2 L}\left(\frac{<k(k-1)>}{<k>}\right)^{L},
$$

where $k$ is the degree sequence of the network and $\langle$. represents the average value of a sequence. We can obtain the expected number of short loops on a specific node as

$$
E\left(C_{L}(i)\right)=\frac{1}{2 L}\left(\frac{a}{b}\right)^{L}-\frac{1}{2 L}\left(\frac{\left(a-k_{i}\left(k_{i}-1\right)\right)\left(b-4 k_{i}\right)}{\left(b-2 k_{i}\right)^{2}}\right)^{L},
$$

where $a=\sum_{h=1}^{N} k_{h}\left(k_{h}-1\right)$ and $b=\sum_{h=1}^{N} k_{h}$.

For directed random networks, the expected number $E\left(N_{L}\right)$ of short loops with length $L$ can be obtained by[10]

$$
E\left(N_{L}\right)=\frac{1}{2 L}\left(\frac{<k_{\text {in }} k_{\text {out }}>}{<k_{\text {in }}>}\right)^{L} .
$$

Like the undirected network, we also deduce a formula to estimate the expected number of short loops on a specific node $i$. The formula of $E\left(C_{L}(i)\right)$ is

$E\left(C_{L}(i)\right)=\frac{1}{L}\left(\frac{c}{d}\right)^{L}-\frac{1}{L}\left(\frac{\left(c-k_{i}^{\text {in }} k_{i}^{\text {out }}\right)\left(1-\frac{k_{i}^{\text {in }}}{d-k_{i}^{\text {out }}}-\frac{k_{i}^{\text {out }}}{d-k_{i}^{\text {in }}}\right)}{d-k_{i}^{\text {in }}-k_{i}^{\text {out }}}\right)^{L}$.

where $c=\sum_{h=1}^{N} k_{h}^{\text {in }} k_{h}^{\text {out }}$ and $d=\sum_{h=1}^{N} k_{h}^{i n}$. To examine the validity of our formula, we calculate the exact short loops number [8] in directed and undirected random networks with prearranged poisson degree sequences and compare them with the expected values from our formulas. The result shows our formulas can perfectly predict the $C_{L}(i)$ of both undirected and directed random networks with given degree sequences. So far, given the degree sequence of directed and undirected networks, we can use these two formulas to predict the loops number on each node in the
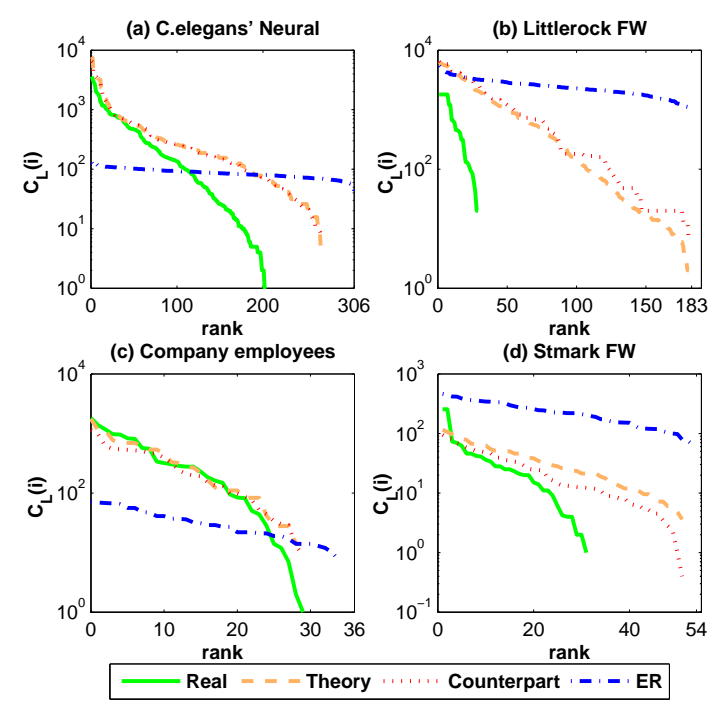

FIG. 1: The zipf plots of $C_{L}^{r}(i), C_{L}^{c}(i), C_{L}^{e}(i)$ and $E\left(C_{L}^{c}(i)\right)$ of four different networks including (a)C.elegans' neural network, (b)Littlerock food web network, (c)High technology company employees' friendship network, (d)Stmark food web network. In this Figure, we use $L=5$ as example. The links for the random networks are averaged by 100 times.

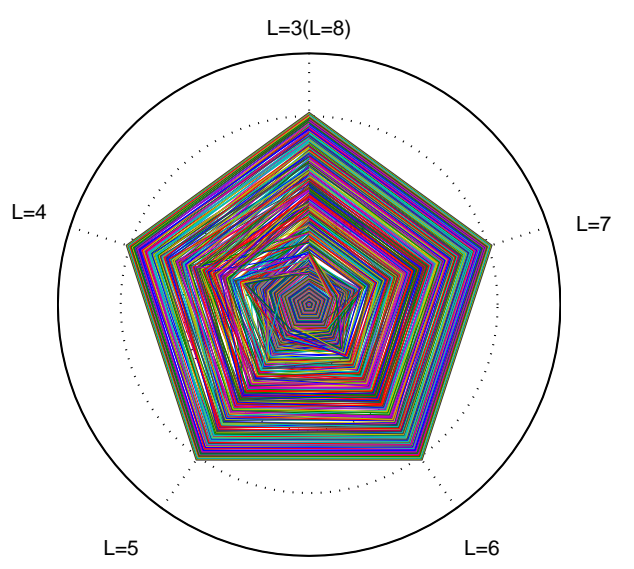

FIG. 2: The rank clocks of $C_{L}(i)$ of C.elegans' neural network, with each axis running from rank 1 on the circumference to 306 at the center. The top rank 1 represents the node with the most loops passing through while the bottom rank 306 is the node with the fewest loops. Each vertex on the circumference stands for the length of the loops.

uncorrelated random networks. That is to say the distribution $C_{L}^{c}(i)$ can be represented by the distribution of $E\left(C_{L}^{c}(i)\right)$ which can be simply calculated by our formulas.

In fig.1, we compare the zipf plots of $C_{L}^{r}(i), C_{L}^{c}(i)$, $C_{L}^{e}(i)$ and $E\left(C_{L}^{c}(i)\right)$ in four different networks including the C.elegans' neural network, the littlerock food web 
network, high technology company employees' friendship network and the stmark food web network. If a node has no loop passing through, $C_{L}(i)=0$. In the zipf plot, this node will not appear in the log axis. Hence, the shorter tail of the line means all the loops are inclined to locate in several specific nodes. In addition, the steeper slope of the line in zipf plots indicates the distribution of $C_{L}(i)$ is more skewed, which means that nodes are quiet different from each other in loops number. If these two features are more significant, the distribution of the $C_{L}(i)$ will be more heterogenous. Comparing the real networks to ER random networks, we find that the loops are more heterogenous in some real networks such as neural networks and some food web networks. Moreover, from the $C_{L}^{r}(i)$ and $C_{L}^{c}(i)$, we can easily find that the degree sequence are not sufficient to describe the heterogeneity of loops distribution on nodes. In many cases, $C_{L}^{r}(i)$ performs a more significant heterogeneity than $E\left(C_{L}^{c}(i)\right)$ and $C_{L}^{c}(i)$. However, loops are distributed in some social networks almost the same as in the counterpart networks. A typical example is given in fig.1(c).

In particular, we study $C_{L}(i)$ with different lengths in the C.elegans' neural networks. In fig.2, we use the rank clocks to test whether the rank of loops on each node varies dramatically with different loops length $L[21]$. In fig.2, the rank clocks show perfect pentagon in the circumference which means that the top ranks of $C_{L}(i)$ do not change. Hence, we can clearly see that although for different length $L$, the heterogenous loops distributions share the same top rank.

From the heterogenous distribution of the loops in each node, we can easily find that loops locate more uneven in some real networks than in counterpart random ones. That is to say, some nodes of the real network are relatively rich in loops while loops are sparse in some other nodes. This phenomenon indicates that besides the total number, the detail organization of these loops in the real networks is quiet different from the counterpart random networks. If these nodes with many loops tightly connect with each other in the same community, of course this community will be extremely rich in loops. Combined with the result in fig.2, the same top rank in the heterogenous loops distribution will enhance the richness in loops in the community. In the following section, we will discuss the phenomenon of uneven loops location by studying the short loops in a specific kind of community of networks.

The rich loop core phenomenon. To investigate the detail organization of the self-avoiding loops in a network, we will study the loops number of different communities. In this paper, we consider that a loop belongs to a community only if all the nodes of the loops are included in this community. For these real networks we are about to analyze in this paper, because long loops are strongly related to the size of a chosen community, short loops are the main elements in the community. Therefore, we only consider short loops in this paper. It has been investigated that many real networks have fewer loops than the

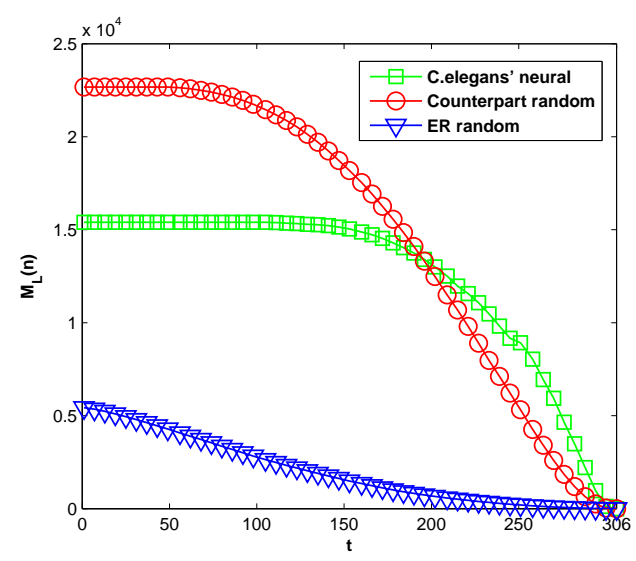

FIG. 3: The change of the loops number as the nodes removing from three kinds of networks. In this figure, we choose $L=5$ as a example. In fact, because the community obtained in this process is the selected community, the loops number in these communities is $M_{L}(n)$. Furthermore, the size of selected community $n=N-t$ in each step $t$. The links for the random networks are averaged by 50 times.

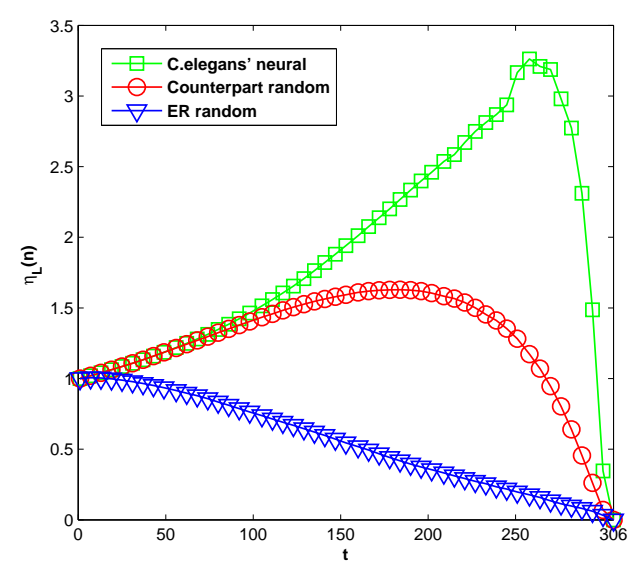

FIG. 4: The loop density ratio $\eta_{L}(n)$ of three network in each step of node removing process. We choose $L=5$ as a example. It is clear that loops locate more unevenly in C.elegans' neural network than the counterpart random networks. Furthermore, the size of selected community $n=N-t$ in each step $t$. The links for the random networks are averaged by 50 times.

randomized counterparts in both short loops and long loops such as C.elegans' neural network, Food Web networks, Power-grid networks etc[10]. Here, we take some of these real networks to make a further study of the loops in their communities.

First, we introduce a node removing process. In each step $t$ of the node removing process, we remove the node with the smallest $C_{L}(i)$ in the network. It means that the node with fewer loops passing through will be removed first. Specifically, the $C_{L}(i)$ should be updated in each step. After $N$ steps, we can remove all the nodes from 

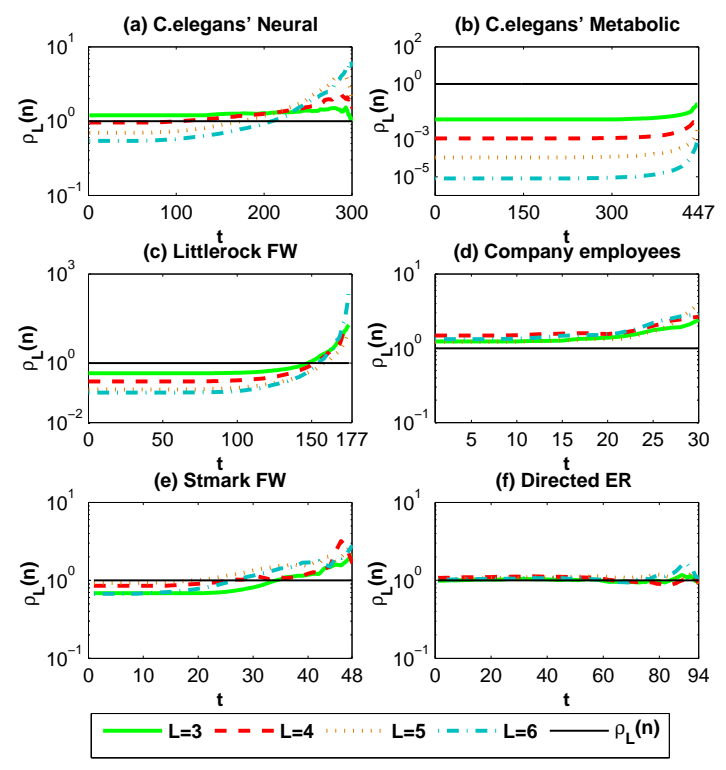

FIG. 5: Typical results of comparing selected communities' loops of many networks to that of the counterpart random models. The results are averaged by 50 times. (a) C.elegans' neural network, (b) C.elegans' metabolic network, (c) and (e) are food web networks in two different places, (d) Company employees' friendship network which is obtained by questionnaire, (f) directed ER random networks with 100 nodes and 800 links.

the network. If we want to obtain a community with size $n$, the number of the removed nodes should be $t=$ $N-n$. Because the node about to be removed in every step has the fewest loops among the remaining nodes, the community obtained by this process has the richest short loops among all the communities with the same size. The community obtained by the node removing process is called the selected community in this paper, the loops number in this community is denoted as $M_{L}(n)$. Obviously, $M_{L}(N)=N_{L}$ and $M_{L}(0)=0$. If a network is greatly uneven in the loop location, $M_{L}(n)$ will decrease slowly in the beginning while decline dramatically in the end through out the node removing process.

We analyze the C.elegans' neural network by the node removing process as an example. In fig.3, we compare the C.elegans' neural network, the counterpart random networks and the corresponding ER random networks. It can be seen that although the counterpart random networks have more short loops than C.elegans' neural network, it can not compete with the C.elegans' neural network in some specific selected communities. For instance, the selected community with 50 nodes in the C.elegans' neural network has more short loops than that in the counterpart random networks as in fig.3.

Furthermore, we define the loop density as the proportion of the loops number and the community size. So we can easily get the loop density of the original network $N_{L} / N$. To compare the loop density of the selected communities and the original network, we define the loop density ratio $\eta_{L}(n)$ in each selected community during the node removing process as

$$
\eta_{L}(n)=\frac{M_{L}(n) N}{N_{L} n}
$$

The loop density ratio $\eta_{L}(n)$ is the proportion of the selected community's loop density and the original network's loop density. If the loops locate unevenly in a network, $\eta_{L}(n)$ will become larger than 1 during the node removing process. It indicates some communities in the network have bigger loop density than the original network. Obviously, in the C.elegans' neural network, some of the selected communities enjoy larger loop density than the original network, so $\eta_{L}(n)$ is larger than 1 as shown in Fig.4. Although the counterpart random networks can also have a $\eta_{L}(n)$ larger than 1 , its value is always lower than that of C.elegans' neural network. This feature indicates that loops locate more unevenly in C.elegans' neural network than in the counterpart random networks, which means that lots of loops are limited in some specific nodes of the C.elegans' neural network.

So the unevenness may result in a rich loop core phenomenon, which means some selected communities with far higher loop density compared with the original networks. Although many real networks have fewer short loops compared to counterpart random models, the rich loop phenomenon will make some communities in real networks more loopy than the corresponding communities in the counterpart random networks. It can be detected by the ratio as:

$$
\rho_{L}(n)=\frac{M_{L}^{r e a l}(n)}{M_{L}^{r a n d}(n)}
$$

where $n$ is the size of the selected community. For example, the index will turn from $\rho_{L}(n)<1$ to $\rho_{L}(n)>1$ as nodes removed in the C.elegans' neural network as show in fig.3. Also, we also investigate many other real networks, some typical results are shown in fig. 4 .

As in fig.4 (a), (c) and (e), although these real networks, such as neural networks and some food web networks, have fewer total loops than the counterpart random networks, some selected communities of them are more loopy than that of counterpart random ones. Moreover, we find that short loops with different lengths $L$ perform the same trend in the rich loop core phenomenon. If the loops with specific length $L$ locate unevenly in the networks, loops with other lengths locate uneven as well. However, not all the networks have this kind of phenomenon as shown in Fig.4(b). Some real networks with far fewer loops than the counterpart random networks have $\rho_{L}(n)<1$ for all $n$. For example, the C.elegans' metabolic network belongs to this category. In addition, we find that some social networks have more short loops than the counterpart random ones as in fig.4(d). These networks are not discussed in ref[10]. This category includes the prisoners' friendship network, high technology 
employees' friendship network, the family visit network, the flying-team partner choosing network, the dining table partner choosing network and so on[22]. If these networks enjoy more uneven loops location, their selected communities can only be more and more loopy than that of counterpart random networks as the community size $n$ varies, see fig.4(d). Finally, we use the index $\rho_{L}(n)$ to detect a ER random network with 100 nodes and 800 links. The result in fig.4(f) shows that $\rho_{L}(n)=1$ approximately.

Besides the rich loop core phenomenon, how to find the rich loop core in a network is an interesting question. In this paper, we simply consider the rich loop core appears at the maximum $\eta_{L}(n)$, which means the rich loop core will have highest loop density than any other community in the networks. Some typical results are shown in fig.6. The littlerock food web and the C.elegans' metabolic network have significant rich loop cores which indicates they have much higher loop density community compared with the original networks. The C.elegans' neural networks and the StMark food web have such rich loop cores as well. On the contrary, this phenomenon is not so obvious in company employees networks and large degree ER random networks. For the C.elegans' metabolic network, the high loop density ratio is due to the small number of total loops. These loops unavoidably locate in several specific nodes, so the loops density in the selected community will be very large compared with the original network. Furthermore, we analyze the rich loop cores of the food web and neural networks. It is interesting that most of the nodes in the cores are from interneurons in C.elegans' neural network and from microorganisms in food web networks. Specifically, the rich loop cores of C.elegans' neural networks have different sizes $n$ for different loop lengths $L$. For example, $n=31$ when $L=3$, $n=38$ when $L=4, n=50$ when $L=5$ and $n=51$ when $L=6$. These rich loop cores share 30 nodes and 24 of them are interneurons. The C.elegans' neural networks are composed by sensory neurons, interneurons and motor neurons. Most interneurons are in the nerve ring ganglia. Their main function is to process signals [23,24]. So the loops number in these interneurons is relatively larger than the others. Likewise, the rich loop cores in stmark food web networks are $n=20$ when $L=3, n=22$ when $L=4, n=24$ when $L=5$ and $n=24$ when $L=6$. These rich loop cores share 14 nodes and 10 of them are microorganisms. In the food web networks, the microorganisms are the main element in microbial loops which are strongly related to fixed carbon repacking and recovery path of ecosystem[19].

Measurement for unevenness of loop location. In order to quantify how unevenly loops locate in the networks, we present an index which bases on the node removing process. In order to Simplify the computing complexity, we do not update $C_{L}(i)$ in each step during the node removing process in this section. We test and find the result obtained in way is sufficient to represent that by updating $C_{L}(i)$ in each step statistically.
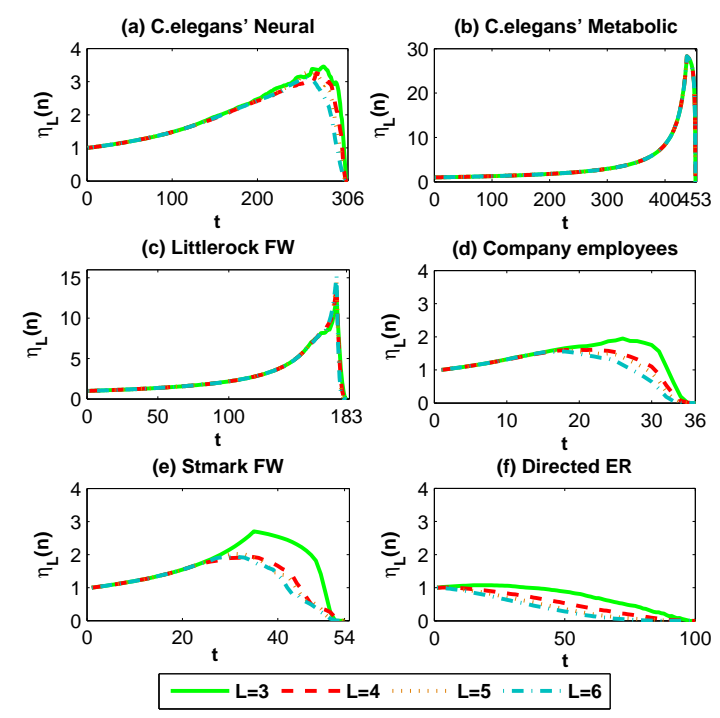

FIG. 6: The rich loop core phenomenon of some typical real networks. The links for the random networks are averaged by 50 times. (a) C.elegans' neural network, (b) C.elegans' metabolic network, (c) and (e) are food web networks in two different places, (d) Company employees' friendship network which is obtained by questionnaire, (f) directed ER random networks with 100 nodes and 800 links.

Moreover, we remove the nodes based on the attacking rate $p$. For example, if $p=0$, no node is removed and the network is all the same with the original network and the loop number is $N_{L}$. If $p=0.1$, we just remove $[p N]_{\text {ceil }}$ nodes from the network and the loop number is $M_{L}\left(N-[p N]_{\text {ceil }}\right)$. Here, $[.]_{\text {ceil }}$ represents the operation of rounding upward. Then, we use $A_{L}(p)=\frac{M_{L}\left(N-[p N]_{c e i l}\right)}{N_{L}}$ to normalize the loop number of each community so that $A_{L}(p)$ which is corresponding to the loop number declines from 1 to 0 through out the node removing process as shown in fig.7. Again, we use $L=5$ as an example in fig.7.

If a network is significantly uneven in the loop location, $A_{L}(p)$ will decline slowly in the beginning while dramatically in the end during the node removing process. On the contrary, if loops locate evenly in the network, the $A_{L}(p)$ will decline almost the same as the corresponding ER random networks. Therefore, the unevenness of loop location can be measured by the difference between the real network and the corresponding ER random network. Here, we use $I_{L}^{r}(p)=A_{L}^{r}(p)-A_{L}^{e}(p)$ to estimate the difference. So the unevenness of loops location can be represented by the index as

$$
R_{L}=\int_{0}^{1} I_{L}(p) d p
$$

where $-1<R_{L}<1$. The severer the unevenness is, the larger the index $R_{L}$ is, which means the loop location departs more largely from the corresponding ER random network. Of course, the index $R_{L}$ can also be used in 

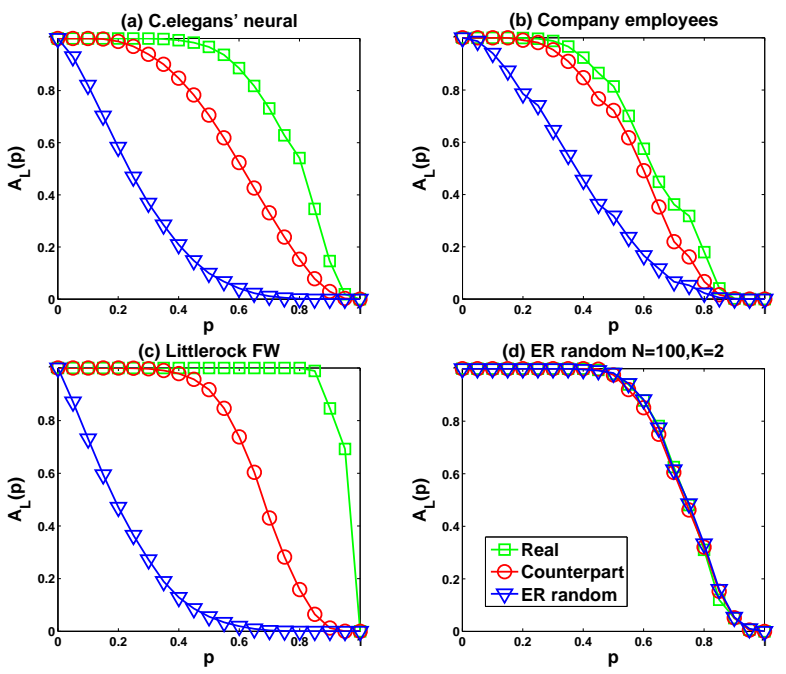

FIG. 7: the $A_{L}^{r}(p), A_{L}^{c}(p)$ and $A_{L}^{e}(p)$ change as the $p$ in different networks including C.elegans' neural network, high technology company employees' friendship network, littlerock FW network, and ER random network with small degree. $L$ is chosen as 5 and the result is averaged by 50 times.

analyzing the counterpart random network.

Actually, the index $R_{L}$ is the area between the lines of the real network and the ER random network in fig.7. It can be seen that the real networks and the counterpart random networks can be different in the unevenness, as the index $R_{L}^{r} \neq R_{L}^{c}$. Typically, if the real network is very sparse in the links, the network has only small number of loops. This will lead to a phenomenon that some part of the network has some loops, while other part has no loop at all. However, the corresponding ER random network has the same condition too. For the $R_{L}$ is the area between the lines of the real network and the ER random network, the $R_{L}$ will be a small value under this circumstance. Whether this uneven location of loops results from the specific structure of the real network or from the small degree as the ER random network can be estimated from the index $R_{L}$. As the figure(d) in the fig.7, we use the index $R_{L}$ to investigate a ER random network with small degree as $K=2$. The result shows that the three lines are almost the same meaning that $R_{L} \approx 0$.

Additionally, we consider several more directed real networks[22]. As mentioned above, although each network has different kinds of short loops based on length $L$, these loops perform almost the same. We use average $R_{L}$ to represent the unevenness in loops location. It can be gained by $\bar{R}=<R_{L}>$ and the $L=3,4, \ldots, L_{\max }$ where $L_{\max }=8$ according to $\operatorname{Ref}[9]$. The index $R$ for these real networks are given in table 1 . From the $\bar{R}_{r}$, how significant the unevenness in loops location is can be known. By comparing the $\bar{R}_{r}$ and the $\bar{R}_{c}$, we can distinguish whether this unevenness results from the degree sequence. In table 1 , it can be seen from the $\bar{R}_{r}$ that the C.elegans neural network, C.elegans metabolic
TABLE I: Results of the analysis of networks based on index $\bar{R}$

\begin{tabular}{lccclc}
\hline network & size & links & $\bar{R}_{r}$ & $\bar{R}_{c}$ & $\bar{R}_{r}-\bar{R}_{c}$ \\
\hline C.elegans' neural & 306 & 2359 & 0.436 & 0.317 & 0.119 \\
C.elegans' metabolic & 453 & 2040 & 0.468 & 0.337 & 0.131 \\
E.coli's metabolic & 896 & 958 & -0.021 & -0.002 & -0.019 \\
Mondego FW & 46 & 400 & 0.231 & 0.257 & -0.026 \\
Michigan FW & 39 & 221 & 0.194 & 0.207 & -0.013 \\
Littlerock FW & 183 & 2494 & 0.672 & 0.439 & 0.233 \\
StMarks FW & 54 & 356 & 0.378 & 0.256 & 0.122 \\
Prisoners & 67 & 182 & 0.169 & 0.004 & 0.165 \\
Flying-teamers & 48 & 351 & 0.108 & 0.060 & 0.048 \\
Company employees & 36 & 147 & 0.219 & 0.163 & 0.056 \\
ER random & -- & -- & 0 & 0 & 0 \\
\hline
\end{tabular}

network and some food web networks are really uneven in loop location. The social networks, Escherichia Coli's metabolic network and some other food web networks do not have such significant unevenness. In fact, the number of different species will affect the loop location in food web networks. For example, too many microorganisms will make the loops more even and too many metazoans will reduce the total loops number, at this time the rich loop core will be more obvious. Additionally, both the C.elegans' metabolic network and the Escherichia Coli's metabolic network have very few loops, but the degree sequence and the total links of the former one allows the counterpart random and the ER random networks to have much more loops while the latter one does not. Hence, the $\bar{R}_{r}$ of these two metabolic networks are different. Moreover, comparing the $\bar{R}_{r}$ and the $\bar{R}_{c}$ in table 1 , it can be found that the degree sequence is not sufficient to describe the unevenness in loops location. It is clear that the C.elegans' neural network, littlerock and stmark food web networks are more uneven in loops location than the counterpart networks. That is why they can have some communities more loopy than the corresponding communities in the counterpart random models despite the fewer total loops number.

Conclusion. The previous works on the loops mainly focus on the total number of loops and the dynamic effect of the loop structure. However, the loop location is also very important in networks. Generally, loops tend to locate in some specific nodes in some real networks, which means some communities of the network are extremely rich in loops while the loops are relatively sparse in other parts. If this uneven location is significant enough, the rich loop core phenomenon can be formed in some real networks.

The rich loop core phenomenon is meaningful for the typical function of real networks. For instance, the loop structure is strongly related to the self-sustained activ- 
ities in neural networks, so the rich loop core may help to understand the functional regions in the neural networks. For the food web networks, almost all the loops in rich loop cores of food web networks are microbial loops and plays an important part in fixed carbon repacking and recovery path of ecosystem. In addition, this uneven location of loops may provide a new way to study the community detection in directed networks, which asks for further research.

Acknowledgement. The authors would like to thank Dong Zhou, Hongzhi You, Prof. Ying Fan and Prof. Yiming Ding for many useful suggestions. This work is supported by NSFC under Grants No. 70974084, No. 60534080 and No. 70771011.
[1] R. Pastor-Satorras, A. Vazquez, A. Vespignani, Phys. Rev. Lett. 87, 258701 (2001).

[2] S. N. Dorogovtsev et al., Rev. Mod. Phys. 80, 1275 (2008).

[3] B. Karrer and M. E. J. Newman, Phys. Rev. Lett. 102, 128701 (2009)

[4] A.Zeng, Y.Hu and Z.Di, Europhys. Lett. 87, 48002 (2009)

[5] K. Klemm and P. F. Stadler, Phys. Rev. E 73, 025101(R) (2006)

[6] E. Marinari, G. Semerjian and V. VanKerrebroeck, Phys. Rev. E 75, 066708 (2007)

[7] E. Marinari, R. Monasson and G. Semerjian, Europhys. Lett. 73, 8-14 (2006)

[8] G. Bianconi, Eur. Phys. J. B 38, 223C230 (2004)

[9] G. Bianconi and N. Gulbahce, J. Phys. A: Math. Theor. 41, 224003 (2008)

[10] G. Bianconi, N. Gulbahce and A. E. Motter, Phys. Rev. Lett. 100, 118701 (2008)

[11] H. D. Rozenfeld et al., J. Phys. A: Math. Gen. 38, 4589C4595 (2005)

[12] A. Roxin, H. Riecke and S. A. Solla, Phys. Rev. Lett. 92, 198101 (2004)

[13] X. Liao et al., arXiv:0906.2356 1

[14] L. F. Lago-Fernandez, R. Huerta, F. Corbacho, J. A.
Siguenza, Phys. Rev. Lett. 84, 2758-2761 (2000)

[15] X. Ma,L. Huang, Y. C. Lai and Z. Zheng, Phys. Rev. E 79, 056106 (2009)

[16] A. Arenas, A. Daz-Guilera, J. Kurths, Y. Moreno and C. Zhou, Physics Reports 469, 93-153 (2008)

[17] J.D. Noh, Eur. Phys. J. B 66, 251-257 (2008)

[18] V. VanKerrebroeck and E. Marinari, Phys. Rev. Lett. 101, 098701 (2008)

[19] E. Sherr and B. Sherr, Limnology and Oceanography, 33, 1225-1227 (1988)

[20] Z. Burda, J. Jurkiewicz, A. Krzywicki, Phys. Rev. E 70, 026106 (2004)

[21] M. Batty, Nature 444, 592-596 (2006)

[22] The network datas are available on line at http://vlado.fmf.unilj.si/pub/networks/data/, http://www.imsc.res.in/ sitabhra/research/neural/celegans/, http://www.casos.cs.cmu.edu/index.php, www.cosinproject.org/.

[23] J. G. White, E. Southgate, J. N. Thomson and S. Brenner, Philos Trans R Soc London B, Biol Sci 314, 1-340 (1986)

[24] E. L. Tsalik, O. Hobert, J Neurobiol 56(2),178-97 (2003) 